\title{
ABUNDANCES IN MAGNETIC Ap STARS
}

\author{
CHARLES R. COWLEY \\ Department of Astronomy, University of Michigan \\ Ann Arbor, MI 48109-1090 USA \\ COWLEY@141.211.104.2
}

\begin{abstract}
The spatial inhomogeneities, Zeeman broadening, and extreme abundance anomalies have thus far prevented definitive analyses of magnetic CP stars. Nevertheless, the abundance anomalies are so large that many of them have been known for decades. Abundance excesses of iron-peak elements of factors of 10 to 100 are common. Relative abundances on the iron peak are not constant. The lines of vanadium and nickel are often weak, and these elements may even be deficient in some stars. In spitè of the large variations, the odd-even effect persists; there is only minor evidence that chemical separation has perturbed the nuclear pattern. The lanthanide rare earths can have excesses of 100 to 1000 or even more in extreme cases. For these elements there is some evidence of fractionation. The actinide rare earth elements uranium and thorium are weakly (but surely!) present in a few of the magnetic CP stars: the best case is HR 465 .
\end{abstract}

\section{INTRODUCTION}

Spectral peculiarities of what we now call magnetic CP stars have been known for nearly a century. The simplest interpretation of these peculiarities requires the stellar atmospheres to have unusual chemical abundances. This is the most widely held view today. In the 1960's the abundance anomalies were thought to originate in unusual patterns of nucleosynthesis. In part, the schemes involving nucleosynthesis arose because the ideas themselves were relatively new, and people were trying to push them as far as they could.

Since Michaud's (1970) initial study, various schemes involving chemical separation have been thought to be much more likely than any involving nuclear reactions. The great appeal of mechanisms involving diffusion is that they are capable of producing quite extreme anomalies in unevolved objects. At the present time, we are convinced that processes that induce chemical separation, or to use a word from geochemistry, differentiation, are relevant to the chemistry of magnetic stars.

The present goal of the study of the chemistry of CP stars is no longer to see if the hypothesis of chemical separation is valid. It is to use the theory to probe the current state and history of these stars. Within this framework, the nuclear pattern is still relevant.

The chemistry of cosmical materials is the result of natural, timedependent processes. Conversely, the history of materials may often be read from their abundance patterns. The nuclear history of the solar system abundance distribution was beautifully interpreted by Burbidge et al. (1957, $\mathrm{B}^{2} \mathrm{FH}$ ) and Cameron (1957). Possibly the most obvious feature of this pattern is the odd-even effect, which is enhanced when abundances are displayed as a function of $\mathrm{Z}$. The basis of this alternation is so obvious to cosmochemists that they often smooth it out, as $\mathrm{B}^{2} \mathrm{FH}$ did in their Fig. I,1. 
Most astronomers thought of nucleosynthesis in terms of the $\mathrm{B}^{2} \mathrm{FH}$ processes, and it took a while for us focus on the odd-even effect. At the present time, this concept provides a particularly useful way in which to view theories involving chemical differentiation and nucleosynthesis. For example, we became convinced that chemical differentiation was relevant to abundances in Ap stars when it became almost undeniable that the odd-even effect was violated at $\mathrm{Mn}$ and $\mathrm{Y}$ in the manganese stars.

In the magnetic stars, violations of the odd-even effect are more difficult to be confident of. Nevertheless, the fact that chemical differentiation is operating in one subset of the CP stars is a strong indication that it operates is others. This point has been often emphasized by Michaud. Possibly the nuclear, odd-even pattern is broken in certain of the magnetic CP stars at europium.

The stars 73 Dra and $\beta \mathrm{CrB}$ may have what we have called Type II odd- $Z$ anomalies. These are cases where an odd- $Z$ element has an abundance higher than either of it's even- $Z$ neighbors. We know of only one case among the "cosmical" abundances (Anders and Grevesse 1989, henceforth, SAD) of a Type II anomaly, and this occurs at beryllium. Here, we have a Type II even- $Z$ anomaly. Before we discuss such matters, let us summarize what we think we know about the abundances in these stars.

\section{Abundances}

General Remarks

Abundances in magnetic CP stars are subject to great uncertainties for a number of reasons. It is well known that in some of these stars, the abundances vary over the stellar surfaces. In addition, the Zeeman effect enormously complicates the theoretical reproduction of stellar spectra. We shall leave elaboration on these matters to experts, and only comment on their results as they seem relevant to abundances per se. Most of the abundances in magnetic CP stars have been based on models with homogeneous surfaces. Numerical experiments need to be done to see just how much error is introduced through the use of such models. Certainly, generalizations are difficult to make because the amount of inhomogeneity varies from one star to another.

The few studies that have tried to account for abundance patches confirm, at least qualitatively, the results from more simplistic models (e.g. Landstreet et al. 1989).

Few people have had the courage to do abundance work in these stars in recent years. Most of the work been wisely restricted to the more tractable nonmagnetic objects. Certainly the mercury and platinum anomalies in manganese stars rival-in orders of magnitude-those of their magnetic congeners. Nevertheless, the overall chemical peculiarities of the magnetic stars approaches a qualitative difference, because of the overall increase in complexity.

\section{Reviews}

There are quite a number of useful summaries of spectra and abundances in these stars. We mention those of Preston (1974), Hack (1975), 
Khokhlova (1985), and Ryabchikova (1991). We often refer to the Jaschek's (1974) comparative description of the spectra. It might seem that almost anything one would want to know about an A star was contained in the very fine book by Wolff (1983). She even has a section on $\lambda$ Boo stars, although not the latest ideas, concerning fractionation of interstellar material.

$\underline{\text { Results }}$

All of the reviews of abundances say similar things-large enhancements of various elements, and depletions of a few others. Unfortunately, apart from a few people who really spend a lot of time looking at the spectra, it may seem that these people all say the same thing, or what is worse, that the stars within the different peculiarity classes are similar. To those really familiar with the spectra, some stars within a given class are as different from one another as they are from stars in a different class.

The light elements were reviewed by Ryabchikova (1991), and will not be discussed here, apart from the comprehensive study of the oxygen abundances in CP and normal stars by groups in France and Italy (Faraggiana et al. 1988, Gerbaldi et al. 1989, and van't Veer-Menneret et al. 1989). Quite a large number of stars were studied, and results were rewarding. A plot of the abundances of oxygen vs. effective temperature revealed a very nice separation of the different peculiarity types.

Geochemists would call such a plot a variation diagram. These are used in petrology to discover evolutionary relationships as well as to exclude them. Isolated groups of points on such diagrams are taken to imply distinct origins. Real, physical mechanisms can cause the groups to merge, both in the stellar and the geochemical situations. But it is difficult to separate merging caused by physical or chemical processes from those resulting from analytical errors.

Overabundances of some iron-peak elements of factors of 10 to 100 are found in magnetic CP stars. This is the situation for chromium, manganese, and iron itself, while larger excesses of strontium and the lanthanides can occur. For the latter elements, the figures given by Mrs. Hack, factors of 100 to $>1000$ remain useful to keep in mind.

Adelman's monumental dissertation work has never been superseded. No one else, even Saul himself has had the courage or strength to repeat what he did some 20 years ago. In the recent decade, Ryabchikova and her colleagues (see below) have studied a number of these stars. Specialized studies have been made of a few elements (e.g. Sadakane and Okyudo 1989, Roby and Lambert 1990). Abundance information is also available from groups mapping the surface inhomogeneities. (e.g. Rice and Wehlau 1991). The results are in general agreement, but certainly not in the details. Adelman stressed how different these objects were from one another. His work supported the findings of Bidelman (1966, 1968), though again, there were differences.

Among the lanthanide elements, Bidelman's qualitative assessments were actually better than Adelman's quantitative ones. We now understand why this was. The quantitative determinations were often based 
on a few features that turned out to be badly blended. The features were in fact dominated by what Johansson and Cowley (1988) call second generation lines. This essentially means lines that are not in the Multiplet Tables. A few critical blends badly distorted the determinations of certain lanthanides. Most of the blends were caused by second generation chromium lines.

Unfortunately, the situation now is that we really cannot be sure what is going on with the lanthanide pattern in the magnetic stars. Things are much clearer in the Am stars, where very fine work has ben done by van't Veer-Menneret, Burkhart, and Coupry (1988). These workers found a nuclear pattern, but not a solar one in HR 178. The magnetic star $\gamma$ Equ has a rare earth pattern that is similar to that of the Am stars (Magazzu and Cowley 1986, Cowley 1976). However, in certain other magnetic stars, notable peculiarities occur. We mentioned already in 1975 the troughs that appear in the lanthanide patterns at $\mathrm{Nd}$ and $\mathrm{Sm}$ in the stars $\beta \mathrm{CrB}$ and HR 7575 . James Sowell and I have some preliminary results, alas, still unpublished, that confirm the presence of a Nd hole in $10 \mathrm{Aql}$. The depth of the hole appears not much greater than the credibility threshold-about a factor of 2 or 3 . I think it is correct because it is supported by WCS.

Coincidence statistics tell us that $\mathrm{Sm}$ is strong in $10 \mathrm{Aql}$, but this remains to be confirmed quantitatively.

\section{The Iron Peak}

Spectroscopists often refer to the elements whose neutral atoms are first filling the $3 \mathrm{~d}$ shell as the iron group. We shall be more concerned here with the iron-peak elements. The latter name arises from nucleosynthesis. SAD abundances of iron peak elements, essentially chromium through nickel, could be fit reasonably well by nuclear statistical equilibrium.

Among the iron-peak elements, where more lines, and more secure identifications were available, the results were markedly better. By this, we mean that the quantitative results were in better agreement with the "visual spectroscopy," as the Jaschek's have used this term, or WCS. These iron-group elements show a remarkable pattern. While the abundances show a wide variation, the nuclear, odd-even effect somehow manages to persist. This is plain in Figure 2 of Adelman (1973), and also in Fig 1 of Ryabchikova and Ptitsyn (1986).

Our Figure 1 uses the determinations of Ryabchikova and Ptitsyn, but with slightly different weighting. The general pattern found by Adelman (1973) persists.

The persistence of the odd-even pattern in this way can not be taken as a proof that the abundance anomalies arose through nuclear processing. Having said this, it is surely worth wondering why nonnuclear processes have produced both a highly non-solar pattern and one that resembles the results of nucleosynthesis. We have no doubt that chemical separation can affect the patterns of the iron-group elements, because it has produced the nonnuclear odd- $\mathrm{Z}$ anomaly in the mercury manganese stars. What we see in the magnetic stars is a quite different pattern. Generally, all of the iron group elements have been enhanced, and by quite 
large factors. Manganese itself, which we would expect to be pushed up is indeed enhanced, but not by so much as the even- $Z$ element, chromium. The manganese abundance varies quite a bit in these stars, and we have no trouble at all attributing some of this variation to chemical differentiation.

Iron Group Abundances

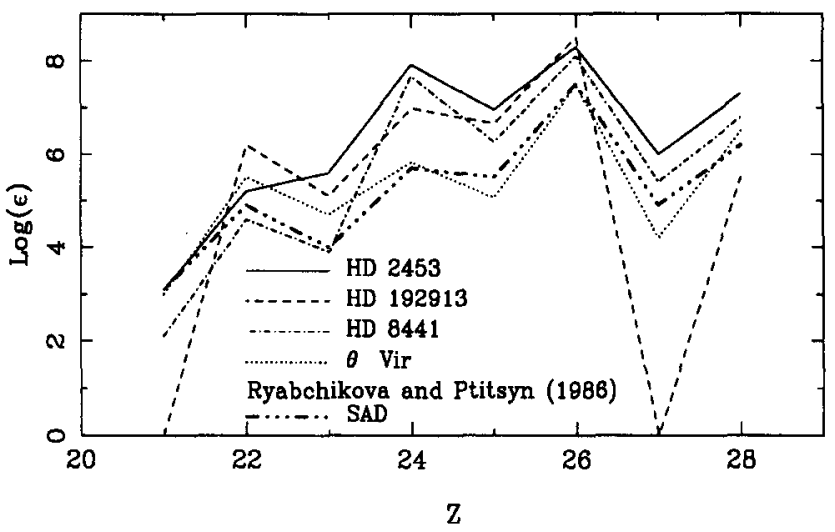

Figure 1: Abundances in 3 Magnetic Ap stars, and the "normal star" $\theta$ Vir (dotted). The SAD is shown with a heavy dash-dot-dot-dot. The solid line is HR 2453, and the dot-dash shows HD 8441. Both are cool Ap stars studied by Adelman. The dash shows the silicon star HR 192913. Missing abundances are arbitrarily assigned $\log (\epsilon)=0.0$.

Is it possible that some of the magnetic Ap stars have formed from material that has been enriched in refractory elements as a result of gasgrain separation in the interstellar medium? What sort of abundance pattern would be expected? Most of the relevant calculations have been done in connection with the formation of the planets (cf. Lewis and Prinn 1984). This is a very broad field with a large literature, and we confine ourselves to a few remarks. The most refractory elements in the solar system calculations are $\mathrm{Ca}, \mathrm{Al}$, and $\mathrm{Ti}$, which form the oxides perovskite, corundum, and ilmenite. At the temperatures and pressures relevant to the solar system, iron-nickel alloys condense a little sooner than the more common silicates.

Abundances of these elements need to be carefully investigated to see if they give any indication that the magnetic CP's formed from grain-rich material.

\section{High Iron Abundance and a Few Other Strange Things}

Quite a number of abundance determinations in magnetic Ap's have shown values of $[\mathrm{Fe} / \mathrm{H}]$ near -3.0. At first, we didn't believe them, because our surveys with wavelength coincidence statistics (WCS) did not 
show large differences in the results for iron from one star to another (Cowley and Aikman 1980). However, we now think this was because our line lists did not cover a wide enough range of intensities.

We give a short list of magnetic Ap stars below for which the iron abundance may be higher than current ideas can explain. It is based on our own surveys as well as the references cited. In addition, we call attention to a few other peculiarities that have shown up in our WCS surveys. Quantitative investigations should be rewarding. The designation HR 465(A) refers to the rare earth maximum of this most unusual object. HR 465(B) refers to a spectrum obtained in 1974 (cf. Cowley 1976).

We recommend these stars to analytical spectroscopists for careful, modern analyses.

Table 1. Stars with Selected Spectral Peculiarities

\begin{tabular}{|l|l|}
\hline Star & Remarks \\
\hline HR 465(B) & Fe II, III; (Mo II) \\
HR 6870 & high iron (needs modern, low noise study) \\
HR 4816 & Fe II strong, Fe III \\
HR 7575 & Fe II; Mn I, II strong;Mo II cool Ap; needs work \\
HD 2453 & Fe II strong, Fe III; Mo II \\
HD 8441 & WCS => more Fe than quant. analyses (??) \\
HD 135679 & Fe II strong, Fe III Gulliver \& MacRae 1975 \\
B CrB & Mo II, like HR 7575 but weaker manganese. Nd-Sm hole \\
HR 465(A) & Mo II and Nb II ; Lanthanides!!; Th II,III; U II \\
HR 4854 & Pt II pres., Mn II v. str. Eu II v. weak; reln. to Hg-Mn?? \\
HR 710 & Th II? Cool Ap strong Na I, needs analysis \\
HD 51418 & Nb II Ho III strong \\
HD 101065 & Nb II Przybylski's lanthanide star \\
&
\end{tabular}

In the last five years or so, we have begun to use two lists containing faint Fe II lines, one from Kurucz and Peytremann (1975), and another from Johansson (cf. Johansson 1988) with $5 p-6 d$ transitions. The $5 p$ levels of Fe II start at some $89,000 \mathrm{~cm}^{-1}$ or some $11.0 \mathrm{eV}$ up.

In addition to the new Fe II lines, we recently created a list of Fe III lines from Kurucz (1991) that allowed us to identify this spectrum in a number of stars, including some of Adelman's cool Ap stars as well as a few mercury manganese stars. In the latter case, it is comforting that we have made the identifications in iron-rich stars, such as HR 7664 or 112 Her. This list is surely superior to the one we had used for more than a decade, based on the analysis by Glad (1956), and this is surely because of the non-thermal nature of Glad's source. 
Our newer WCS surveys support recent results giving iron abundances, at least in some patches, in some stars, as high as $\mathbf{- 3 . 0}$ (cf. Landstreet, et al, 1989, Rice and Wehlau 1991). We recommend that workers try to include Fe III in their analyses in order to strengthen the overall reliability of these findings. Muthsam and Cowley (1984) obtained a mean abundance for Fe I, II, and III near -3.0 for HR $6870=$ HD 168733; Ryabchikova and Piskunov (1986) got -3.7 from Fe II.

We are unaware of any theoretical basis for abundances of iron that can reach as high as -3.0 . The old calculations of Michaud et al. (1976) show that iron should sink, and this seems to be the case in 53 Tau, for example. The magnetic Ap's by contrast, show the mirror image of the Michaud et al. predictions.

Clearly this situation deserves careful scrutiny.

We wish to thank Director Jackson, Dr. W. W. Weiss, and the staff of the Vienna Observatory for their hospitality during the time this manuscript was being prepared. We thank Drs. T. Ryabchikova and N. Piskunov for many useful conversations and help of various kinds relevant to the preparation of this manuscript.

\section{References}

Adelman, S. J. 1973, Ap. J. Suppl, 26, 1.

Anders, E., and Grevesse, N. 1989, Geochim. Cosmochim. Acta., 53, 197.

Bidelman, W. P. 1966, in Abundance Determinations in Stellar Spectra, IAU Symposium, 26, ed. H. Hubenet (London: Academic Press), p. 229 .

Bidelman, W. P. 1968, in Nucleosynthesis, ed. W. D. Arnett, C. J. Hansen, J. W. Truran, and A. G. W. Cameron (New York: Gordon and Breach), p. 63.

Burbidge, G. R., Burbidge, E. M., Fowler, W. A., and Hoyle, F. 1957, Rev. Mod. Phys., $29,547\left(\mathrm{~B}^{2} \mathrm{FH}\right)$.

Cameron, A. G. W. 1957, Pub. Astron. Soc. Pac., 69, 201.

Cowley, C. R. 1976, Ap. J. Suppl., 32, 631.

Cowley, C. R., and Aikman, G. C. L. 1980, Ap. J., 242, 684.

Faraggiana, R., Gerbaldi, M., Van't Veer, C., and Floquet, M. 1988, Astron. Ap., 201, 259.

Gerbaldi, M., Floquet, M., Faraggiana, R., and Van't Veer-Menneret, C. 1989, Astron. Ap. Suppl., 81, 127.

Glad, S. 1956, Ark. Fys., 10, 21.

Gulliver, A. F. and MacRae, D. A. 1975, Astron. J., 80, 402.

Hack, M. 1975, in Physics of Ap-Stars, ed. W. W. Weiss, H. Jenkner, and H. J. Wood (Vienna: Universitatssternwarte), 255.

Jaschek, M., and Jaschek, C. 1974, Vistas in Astron., 16, 131.

Johansson, S. 1988, in Physics of Formation of Fe II Lines Outside LTE, ed. R. Viotti, A. Vittone, and M. Friedjung (Dordrecht: D. Reidel), p. 13.

Johansson, S., and Cowley, C. R. 1988, J. Opt. Soc. Am., 5B, 2264.

Khokhlova, V. L. 1985, Sov. Sci. Rev. E., Ap. Space. Phys., 4, 99.

Kurucz, R. L. 1991, in Precision Photometry: Astrophysics of the Galaxy, ed. A. G. D. Phillip, A. R. Upgren, and K. A. Janes. (Schenectady: L. Davis Press), p. 27.

Kurucz, R. L., and Peytremann, E. 1975, Smith. Ap. Obs. Spec. Rept., 362. 
Landstreet, J. D., Barker, P. K., Bohlander, D. A., and Jewison, M. S. 1989, Ap. J., 344, 876.

Lewis, J.S., and Prinn, R. G. 1984, Planets and their Atmospheres, (Orlando: Academic Press).

Magazzu, A., and Cowley, C. R. 1986, Ap. J., 308, 254.

Michaud, G. 1970, Ap. J., 160, 641.

Michaud, G., Charland, Y., Vauclair, S., and Vauclair, G. 1976, Ap. J., 210, 447.

Muthsam, H., and Cowley, C. R. 1984, Astron. Ap., 130, 348.

Preston, G. W. 1974, Ann. Rev. Astron. Ap., 12, 257.

Rice, J. B., and Wehlau, W. H. 1991, Astron. Ap., 246, 195.

Roby, S. W., and Lambert, D. L. 1990, Ap. J. Suppl., 73, 67.

Ryabchikova, T. A. 1991, in Evolution of Stars: The Photospheric Abundance Connection, ed. G. Michaud and A. Tutukov (Dordrecht: Kluwer).

Ryabchikova, T. A., and Ptitsyn, D. A. 1986, in Upper Main Sequence Stars with Anomalous Abundances, ed. C. R. Cowley, M. M. Dworetsky, and C. Megessier (Dordrecht: Reidel), p. 319.

Ryabchikova, T. A., and Piskunov, N. E. 1986, in Upper Main Sequence Stars with Anomalous Abundances, ed. C. R. Cowley, M. M. Dworetsky, and C. Megessier (Dordrecht: Reidel), p. 45.

Sadakane, K, and Okyudo, M. 1989, Pub. Astron. Soc. Japan, 41, 1055.

van't Veer-Mennertet, C., Burkhart, C., Coupry, M. F. 1988, Astron. Ap., 203, 123.

van't Veer-Menneret, C., Faraggiana, R., Gerbaldi, M., Castelli, F., Burkhart, C., and Floquet, M. 1989, Astron. Ap., 224, 171.

Wolff, S. C. 1983, The A-Stars: Problems and Perspectives, NASA SP-463. 